\title{
Flexural analysis of cross-ply laminated beams using layerwise trigonometric shear deformation theory
}

\section{Abstract}

In the present work, a layerwise trigonometric shear deformation theory is used for the analysis of two layered (90/0) cross ply laminated simply supported and fixed beams subjected to sinusoidal load. The displacement field of the present theory consists of trigonometric sine function in terms of thickness coordinate to take into account the effect of transverse shear deformation. Theory satisfies the transverse shear stress free boundary conditions at top and bottom surfaces of the beam. This model satisfies the constitutive relationship between shear stress and shear strain in both the layers and the axial displacement compatibility at the interface. Virtual work principle is employed to obtain governing equations and boundary conditions. Closed form solution technique has the limitation of simply supported boundary condition. In the present work general solution technique is developed, which can be used for any type of boundary and loading conditions. The transverse shear stresses are obtained using constitutive relation as well from the use of equilibrium equations. The results of displacements and stresses obtained by present theory are compared with the available results in the literature.

\section{Keywords}

Shear deformation; cross-ply laminated beam; trigonometric shear deformation theory.

\section{INTRODUCTION}

Structural elements made up of fiber reinforced composite material are being used in the aeronautical and aerospace industries as well as in the other fields of modern technology, primarily due to their high strength to weight ratio and stiffness to weight ratios and also due to their anisotropic material properties that can be achieved through variation of the fiber orientation and stacking sequence. The ratio of transverse shear modulus to elastic modulus is low for composites, hence shear deformation effects are more pronounced in the composite beams subjected to transverse loads. Analytical and numerical methods can be employed for the analysis of structural systems composed of laminated composite components.

The classical beam theory developed by Euler-Bernoulli (ETB) is used only for thin beams because this theory has neglected both transverse shear and normal strains. Timoshenko [1] has

\section{Y. M. Ghugal and}

S. B. Shinde*

Department of Applied Mechanics, Government Engineering College,

Aurangabad - 431005, Maharashtra

State, India

Received 26 Fev 2012

In revised form 12 Jun 2012 


\section{Nomenclature}

$b \quad=$ Width of beam

$\bar{D} \quad=$ Modified flexural rigidity coefficient as defined in Appendix

$D_{1}, D_{2}, D_{3}, \bar{D}_{3}=$ Constants as defined in Appendix

$D \quad=$ Flexural rigidity

$E^{(1)}, E^{(2)} \quad=$ Young's moduli of layer 1and layer 2, respectively

$h \quad=$ Depth (i.e. thickness) of beam

$L \quad=$ Span of the beam

$S \quad=$ Aspect ratio (i.e. ratio of span to depth of beam)

$x, y, z \quad=$ Rectangular coordinates

$\alpha \quad=$ Neutral axis coefficient as defined in Appendix

$\bar{u} \quad=$ Non-dimensional axial displacement

$\bar{w} \quad=$ Non-dimensional transverse displacement

$\bar{\sigma}_{x} \quad=$ Non-dimensional axial stress

$\bar{\tau}_{z x}^{C R} \quad=$ Non-dimensional transverse shear stress obtained from the constitutive relationship

$\tau_{z x}^{E E}=$ Non-dimensional transverse shear stress obtained from the equilibrium equations

\section{Abbreviations}

Superscripts

CR Constitutive relationships

EE Equilibrium equations

Acronyms

ETB Elementary theory of beam bending

FSDT First-order shear deformation theory

HSDT Higher order shear deformation theory

FEM Finite element method

LTSDT Layerwise trigonometric shear deformation theory,

HOSTB5 Higher order shear deformation theory

HST Higher order shear deformation theory 
developed a thick beam theory to include the effect of the transverse shear deformation. This theory is widely known as first order shear deformation theory (FSDT). This theory assumes a constant shear strain across the thickness of the beam and requires a shear correction factor. However, this factor is problem dependent which is influenced by boundary conditions, loading conditions and stacking sequence of plies in the laminated beams.

In order to overcome the drawbacks of classical and Timoshenko beam theories, higher order or equivalent shear deformation theories have been developed. Research on analytical and numerical modeling of laminated composites has been very active in order to achieve accurate representation of the actual behavior of this kind of structures. Ghugal and Shimpi [2], Reddy [3] and Kreja [4] provided comprehensive reviews of shear deformation theories for laminated beams and plates including merits and demerits of equivalent single layer and layerwise shear deformation theories.

A higher-order beam model formulated by Kant and Manjunatha [5] does not require any shear correction factor, where the model is based on a non-linear variation of longitudinal displacements through the beam thickness. Soldato [6] presented higher order model for cylindrical bending of cross-ply laminated plate with various boundary conditions subjected to single sinusoidal transverse load. Zenkour [7] has developed higher order shear deformation beam theory. Analytical solution of theory is obtained using the Navier solution for simply supported boundary conditions.

Manjunatha and Kant [8], Maiti and Sinha [9], Vinayak et al. [10] used the equivalent single layer, displacement based, higher-order shear deformation theories (HSDT) in the analysis of symmetric and unsymmetric laminated beams and obtained the results using finite element method. These theories are the special cases of Lo et al [11] higher-order theory.

Park and Lee [12] presented a new laminated plate theory in which the inplane displacements vary exponentially through plate thickness. The results based on this theory are obtained for symmetric / antisymmetric cross-ply, angle-ply and unsymmetric laminates under cylindrical bending. Khdeir and Reddy [13] used the state space concept in conjunction with the Jordan canonical form to solve the governing equations for the bending of cross-ply laminated composite beams. The classical, first-order, second-order and third-order theories have been used in the analysis.

Tahani [14] presented a displacement based layerwise beam theory and applied it to the laminated $(0 / 90$ and 0/90/0) beams subjected to a sinusoidal load. Liu and Li [15] compared different laminate theories based on displacement hypothesis emphasizing the importance of layerwise theories and also presented a series of quasi-layerwise theories. Li and Liu [16] presented results of single-layered, two-layered, three-layered cross-ply laminates for cylindrical bending The theory is layer dependent and the number of degrees of freedom involved is very

high and hence it is computationally complicated. Icardi [17] and Arya [18] presented zig-zag layerwise theories for the analysis of thick laminated beams.

It is proved that layerwise higher-order theories based on assumed displacements for individual layers yield more accurate results for the transverse stress in the flexural analysis of thick laminates. Such theories were developed and used by Li and Liu [16], Reddy and Robbins [19 and Lu and Liu [20]. Catapano et al. [21] presented static analysis of laminated beams via a unified for- 
mulation and obtained results for un-symmetric cross-ply laminated beams using closed form analytical solution. Shimpi and Ghugal [22] developed a simple layerwise trigonometric shear deformation theory which satisfies zero transverse shear stress condition at the top and bottom of the beam. The theory also satisfies the shear stress continuity condition at the interface between the layers. The theory includes a minimum number of displacement variables. A closed form analytical solution is presented to obtain the results for beams with simply supported boundary conditions.

In the present paper a previously developed layerwise theory [22] is used and results are obtained for two layered cross ply laminated beams subjected to sinusoidal load with simply supported and fixed-fixed boundary conditions. A general solution technique is developed which can be applied to beam with any type of loading and boundary conditions.

\section{THEORETICAL FORMULATION}

The variationally correct forms of differential equations and boundary conditions, based on the assumed displacement field, are obtained using the principle of virtual work. The beam under consideration consists of two layers: layer 1 and layer 2 .

Layer $1\left(90^{0}\right.$ layer $)$ occupies the region:

$$
0 \leq x \leq L ;-b / 2 \leq y \leq b / 2 ;-h / 2 \leq z \leq 0
$$

Layer $2\left(0^{0}\right.$ layer $)$ occupies the region:

$$
0 \leq x \leq L ;-b / 2 \leq y \leq b / 2 ; 0 \leq z \leq h / 2
$$

where $x, y, z$ are Cartesian coordinates, $L$ is the length, $b$ is the width and $h$ is the total depth of beam. The beam can have meaningful boundary conditions and loading conditions.

The beam subjected to transverse load and geometry of the cross-section is shown in Fig.1.
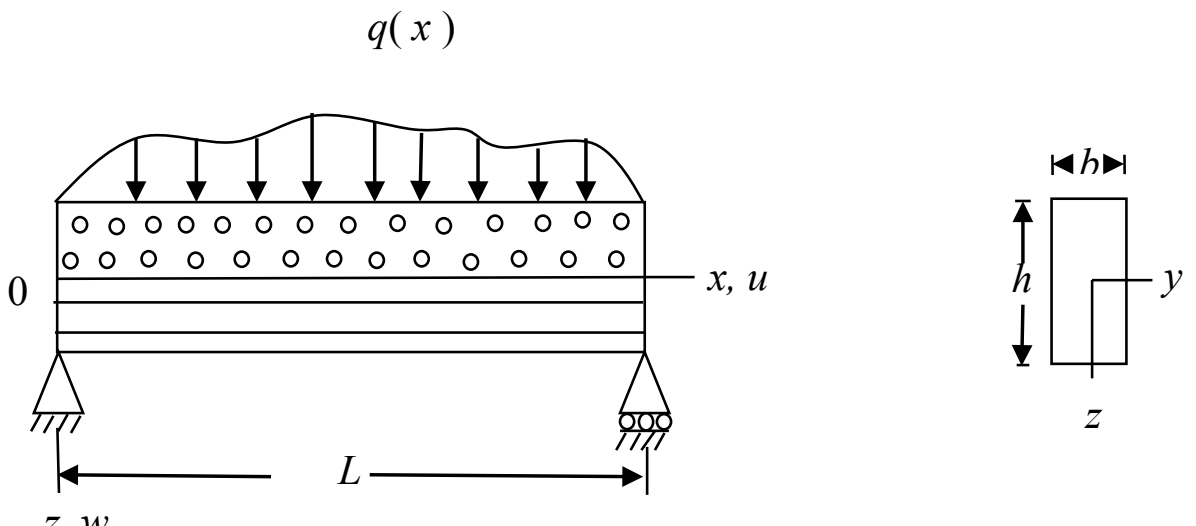

$z, w$

Figure 1 Composite beam with a transverse distributed load and geometry of the cross- section,

Latin American Journal of Solids and Structures 10(2013) $675-705$ 


\subsection{Displacement field}

The displacement field of the present beam theory is of the form [22] as given below:

$$
\begin{gathered}
u^{(1)}(x, z)=-(z-\alpha h) \frac{d w}{d x}+h\left[C_{1}+C_{2} \sin \left(\frac{\pi}{2} \frac{z / h-\alpha}{0.5+\alpha}\right)\right] \phi(x) \\
u^{(2)}(x, z)=-(z-\alpha h) \frac{d w}{d x}+h\left[C_{3}+\sin \left(\frac{\pi}{2} \frac{z / h-\alpha}{0.5-\alpha}\right)\right] \phi(x) \\
w(x, z)=w(x)
\end{gathered}
$$

Here $u^{(1)}$ and $u^{(2)}$ are the axial displacement components in the $x$ direction, superscripts 1 and 2 refer to layer 1 and layer $2 ; w(x)$ is the transverse displacement in the $z$ direction and $C_{1}, C_{2}, C_{3}$ and $\alpha$ are the constants as given in Appendix. The function $\phi(x)$ is a rotation function or the warping function of the cross-section of the beam.

\subsection{Strains}

Normal and transverse shear strains for layer 1 and 2 are as follows:

$$
\begin{gathered}
\varepsilon_{x}^{(1)}=\frac{d u^{(1)}}{d x}=-(z-\alpha h) \frac{d^{2} w}{d x^{2}}+h\left[C_{1}+C_{2} \sin \left(\frac{\pi}{2} \frac{z / h-\alpha}{0.5+\alpha}\right)\right] \frac{d \phi}{d x} \\
\varepsilon_{x}^{(2)}=\frac{d u^{(2)}}{d x}=-(z-\alpha h) \frac{d^{2} w}{d x^{2}}+h\left[C_{3}+\sin \left(\frac{\pi}{2} \frac{z / h-\alpha}{0.5-\alpha}\right)\right] \frac{d \phi}{d x} \\
\gamma_{z x}^{(1)}=\frac{d u^{(1)}}{d z}+\frac{d w}{d x}=\frac{\pi C_{2}}{1+2 \alpha} \cos \left(\frac{\pi}{2} \frac{z / h-\alpha}{0.5+\alpha}\right) \phi \\
\gamma_{z x}^{(2)}=\frac{d u^{(2)}}{d z}+\frac{d w}{d x}=\frac{\pi}{1-2 \alpha} \cos \left(\frac{\pi}{2} \frac{z / h-\alpha}{0.5-\alpha}\right) \phi
\end{gathered}
$$

\subsection{Stresses}

One dimensional constitutive law are used to obtain the normal bending and transverse shear stresses for layer 1 and layer 2 as given below:

$$
\sigma_{x}^{(1)}=E^{(1)} \varepsilon_{x}^{(1)}=E^{(1)}(z-\alpha h) \frac{d^{2} w}{d x^{2}}+E^{(1)} h\left[C_{1}+C_{2} \sin \left(\frac{\pi}{2} \frac{z / h-\alpha}{0.5+\alpha}\right)\right] \frac{d \phi}{d x}
$$




$$
\begin{gathered}
\sigma_{x}^{(2)}=E^{(2)} \varepsilon_{x}^{(2)}=-E^{(2)}(z-\alpha h) \frac{d^{2} w}{d x^{2}}+E^{(2)} h\left[C_{3}+\sin \left(\frac{\pi}{2} \frac{z / h-\alpha}{0.5-\alpha}\right)\right] \frac{d \phi}{d x} \\
\tau_{z x}^{(1)}=G^{(1)} \gamma_{z x}^{(1)}=G^{(1)} \frac{\pi C_{2}}{1+2 \alpha} \cos \left(\frac{\pi}{2} \frac{z / h-\alpha}{0.5+\alpha}\right) \phi \\
\tau_{z x}^{(2)}=G^{(2)} \gamma_{z x}^{(2)}=G^{(2)} \frac{\pi}{1-2 \alpha} \cos \left(\frac{\pi}{2} \frac{z / h-\alpha}{0.5-\alpha}\right) \phi
\end{gathered}
$$

\subsection{Governing Equations and Boundary Conditions}

Using the expressions (6) to (13) for strains and stresses and principle of virtual work, variationally consistent differential equations and boundary conditions for the beam under consideration are obtained. The principle of virtual work applied to beam is as follows:

$$
b \int_{x=0}^{x=L} \int_{z=-h / 2}^{z=0}\left(\sigma_{x}^{(1)} \delta \varepsilon_{x}^{(1)}+\tau_{z x}^{(1)} \delta \gamma_{z x}^{(1)}\right) d x d z+b \int_{x=0}^{x=L} \int_{z=0}^{z=h / 2}\left(\sigma_{x}^{(2)} \delta \varepsilon_{x}^{(2)}+\tau_{z x}^{(2)} \delta \gamma_{z x}^{(2)}\right) d x d z-\int_{x=0}^{x=L} q \delta w d x=0
$$

where the symbol $\delta$ denotes the variational operator. Integrating the Eq. (14) by parts and collecting the coefficients of the primary variables (i.e. $w$ and $\phi$ ), we obtain the governing equations and the associated boundary conditions. The governing equations are as follows:

$$
\begin{gathered}
\frac{d^{4} w}{d x^{4}}-D_{1} \frac{d^{3} \phi}{d x^{3}}-\frac{q}{D}=0 \\
\frac{d w^{3}}{d x^{3}}-D_{2} \frac{d^{2} \phi}{d x^{2}}+D_{3} \phi=0
\end{gathered}
$$

The associated boundary conditions are of the following form:

$$
\begin{aligned}
& \frac{d^{3} w}{d x^{3}}-D_{1} \frac{d^{2} \phi}{d x^{2}}=0 \text { or } w \text { is prescribed } \\
& \frac{d^{2} w}{d x^{2}}-D_{1} \frac{d \phi}{d x}=0 \text { or } \frac{d w}{d x} \text { is prescribed } \\
& \frac{d^{2} w}{d x^{2}}-D_{2} \frac{d \phi}{d x}=0 \quad \text { or } f \quad \text { is prescribed }
\end{aligned}
$$

where $D, D_{1}, D_{2}, D_{3}$ are the constants as defined in Appendix. 
Thus, the static behavior of beam is given by the solution of these variationally consistent governing differential equations and simultaneously satisfaction of the associated boundary conditions.

\subsection{The general solution for static flexure of beams}

Using governing equations (15) and (16); general solution for $w(x)$ and $\phi(x)$ can be obtained. By integrating and rearranging the equation (15), one can get the following equation:

$$
\frac{d^{3} w}{d x^{3}}-D_{1} \frac{d^{2} \phi}{d x^{2}}=\frac{Q(x)}{D}
$$

where $Q(\mathrm{x})=\int q d x+K_{1}$ is the generalized shear force resulting from the support and loading conditions for the beam under consideration. Using Eqs. (16) and (20), a single differential equation in terms of $\phi$ can be obtained as follows:

$$
\frac{d^{2} \phi}{d x^{2}}-\lambda^{2} \phi=\frac{Q(x)}{\eta D}
$$

where the constants $\eta$ and $\lambda$ used in Eq.(21) are given in Appendix. The general solution of above Eq. (21) is given by:

$$
\phi(x)=K_{2} \cosh \lambda x+K_{3} \sinh \lambda x-\frac{Q(x)}{\eta D}
$$

The general solution for transverse displacement $(w)$ can be obtained by substituting the expression for $\phi$ in Eq. (16) and integrating thrice with respect to $x$. The solution is

$$
D w(x)=\iiint \int q d x d x d x d x+\frac{K_{1} x^{3}}{6}+\frac{K_{4} x^{2}}{2}+\frac{D D_{1}}{D_{3}}\left[K_{2} \cosh \lambda x+K_{3} \sinh \lambda x\right]+K_{5} x+K_{6}
$$

where $K_{1}, K_{2}, K_{3}, K_{4}, K_{5}$ and $K_{6}$ are the constants of integration and can be obtained using boundary conditions of the beam under consideration.

\subsection{Axial stress and Transverse shear stresses}

Final solutions for $\phi(x)$ and $w(x)$ can be obtained using Eqs. (22) and (23) depending upon the loading and boundary conditions of the beams. Substituting the final solution for $w(x)$ and $\phi(x)$ in the displacement field, the final displacement can be obtained. Finally, the axial stress $\sigma_{x}$ could be obtained by using stress-strain relationship (constitutive relation) as given in Eqs. (10) 
and (11). The transverse shear stress $\tau_{x z}$ can be obtained either by using the constitutive relation [Eqs. (12) and (13)] or by integrating equilibrium equation with respect to the thickness coordinate. Two-dimensional elasticity equilibrium equations by ignoring body forces can be used to obtain transverse shear stress. The equation is:

$$
\frac{\partial \sigma_{x x}}{\partial x}+\frac{\partial \tau_{x z}}{\partial z}=0
$$

Integrating Eq. (24), with respect to the thickness coordinate $z$ and imposing the following boundary condition at top surface of the beam

$$
\left[\tau_{x z}^{(1)}\right]_{z=-h / 2}=0
$$

Expression for $\tau_{x z}^{(1)}$ can be obtained. To maintain the continuity at interface, another boundary condition is applied, i.e.

$$
\tau_{x z}^{(1)}=\tau_{x z}^{(2)} \text { at } z=0
$$

From this condition expression for $\tau_{x z}^{(2)}$ is obtained for layer 2 which satisfies the requirement of zero transverse shear stress condition on the bottom surface of the beam.

\section{ILLUSTRATIVE EXAMPLES}

To verify the accuracy of the theory two examples of two layered un-symmetric cross ply laminated beams with simply supported and fixed boundary conditions and subjected to sinusoidal loading are analysed. From the analysis the effect of shear deformation and stress concentration on the displacements and stresses is investigated. The material of the beam layers is graphite/epoxy uni-directional composite. The following has been assumed:

$$
\frac{E^{(2)}}{E^{(1)}}=25, \frac{G^{(1)}}{E^{(1)}}=0.20, \frac{G^{(2)}}{E^{(2)}}=0.02
$$

Superscripts (1) and (2) refer to layer-1 and layer-2 respectively.

\subsection{Example1: Simply supported beam with sinusoidal load $q=q_{0} \sin (\pi x / L)$}

A simply supported beam with rectangular cross-section $(b \times h)$ is considered. The beam is subjected to a sine load $q=q_{0} \sin (\pi x / L)$ over the span $L$ at surface $z=-h / 2$ acting in the downward $z$ direction. The associated boundary conditions for this beam are as follows:

Latin American Journal of Solids and Structures 10(2013) $675-705$ 


$$
\begin{gathered}
D \frac{d^{3} w}{d x^{3}}=D \frac{d^{2} \phi}{d x^{2}}=\frac{d w}{d x}=\phi=0 \quad \text { at } \quad x=L / 2 \\
D \frac{d^{2} w}{d x^{2}}=D \frac{d \phi}{d x}=w=0 \quad \text { at } \quad x=0, L
\end{gathered}
$$

The boundary condition, $\phi=0$ at $x=L / 2$ is used from the condition of symmetry of deformation, in which the middle cross-section of the beam must remain plane without warping (Gere and Timoshenko [23]). From the general solution of beam, expression for $\phi$ and $w$ are obtained as follows:

$$
\begin{gathered}
\phi(x)=\frac{q_{0} L}{\pi D D_{3}} \frac{\cos (\pi x / L)}{\Omega} \\
w=\frac{q_{0} L^{4}}{\pi^{4} D} \frac{\chi}{\Omega} \sin \left(\frac{\pi x}{L}\right)
\end{gathered}
$$

where, $\quad \Omega=\left[1+\left(\frac{\pi}{\lambda L}\right)^{2}\right]$ and $\quad \chi=\left[1+\frac{\pi^{2}}{L^{2}} \frac{D_{2}}{D_{3}}\right]$

The expressions for displacements and stresses of the beam are obtained using this solution, which are as follows:

The axial displacement for Layer 1 is expressed as:

$$
u^{(1)}=\frac{q_{0}}{D}\left\{-(z-\alpha h) \frac{L^{3}}{\pi^{3}} \frac{\chi}{\Omega}+\frac{L h}{\pi D_{3} \Omega}\left[C_{1}+C_{2} \sin \left(\frac{\pi}{2} \frac{(z / h-\alpha)}{0.5+\alpha}\right)\right]\right\} \cos \left(\frac{\pi x}{L}\right)
$$

The axial displacement for Layer 2 is expressed as:

$$
u^{(2)}=\frac{q_{0}}{D}\left\{-(z-\alpha h) \frac{L^{3}}{\pi^{3}} \frac{\chi}{\Omega}+\frac{L h}{\pi D_{3} \Omega}\left[C_{3}+\sin \left(\frac{\pi}{2} \frac{(z / h-\alpha)}{0.5-\alpha}\right)\right]\right\} \cos \left(\frac{\pi x}{L}\right)
$$

The axial stress for Layer 1 is expressed as:

$$
\sigma_{x}^{(1)}=\frac{E^{(1)}(z-\alpha h) q_{0} L^{2}}{\pi^{2} D} \frac{\chi}{\Omega} \sin \left(\frac{\pi x}{L}\right)-\frac{E^{(1)} q_{0} h}{D D_{3} \Omega}\left[C_{1}+C_{2} \sin \left(\frac{\pi}{2} \frac{(z / h-\alpha)}{0.5+\alpha}\right)\right] \sin \left(\frac{\pi x}{L}\right)
$$

The axial stress for Layer 2 is expressed as: 


$$
\sigma_{x}^{(2)}=\frac{E^{(2)}(z-\alpha h) q_{0} L^{2}}{\pi^{2} D} \frac{\chi}{\Omega} \sin \left(\frac{\pi x}{L}\right)-\frac{E^{(2)} q_{0} h}{D D_{3} \Omega}\left[C_{3}+\sin \left(\frac{\pi}{2} \frac{(z / h-\alpha)}{0.5-\alpha}\right)\right] \sin \left(\frac{\pi x}{L}\right)
$$

The transverse shear stress using constitutive relationship for Layer 1 is expressed as:

$$
\tau_{z x}^{(1)}=\frac{q_{0} L}{D D_{3} \Omega} \frac{G^{(1)} C_{2}}{(1+2 \alpha)} \cos \left(\frac{\pi}{2} \frac{z / h-\alpha}{0.5+\alpha}\right) \cos \frac{\pi x}{L}
$$

The transverse shear stress using constitutive relationship for Layer 1 is expressed as:

$$
\tau_{z x}^{(2)}=\frac{q_{0} L}{D D_{3} \Omega} \frac{G^{(2)}}{(1-2 \alpha)} \cos \left(\frac{\pi}{2} \frac{z / h-\alpha}{0.5-\alpha}\right) \cos \frac{\pi x}{L}
$$

The transverse shear stress using equilibrium equation for Layer 1 is expressed as:

$$
\tau_{z x}^{(1)}=\frac{q_{0}}{D}\left\{\begin{array}{l}
\frac{E^{(1)} \chi L}{\Omega \pi}\left(\frac{z^{2}}{2}-\alpha h z+\frac{h^{2}}{8}+\frac{\alpha h^{2}}{2}\right) \cos \left(\frac{\pi x}{L}\right) \\
+\frac{E^{(1)} h \pi}{\Omega L D_{3}}\left[z C_{1}+C_{2} \frac{h(1+2 \alpha)}{\pi} \cos \left(\frac{\pi}{2} \frac{(z / h-\alpha)}{0.5+\alpha}\right)+\frac{h C_{1}}{2}\right] \cos \left(\frac{\pi x}{L}\right)
\end{array}\right\}
$$

The transverse shear stress using equilibrium equation for Layer 2 is expressed as:

$$
\tau_{z x}^{(2)}=\frac{q_{0}}{D}\left\{\begin{array}{l}
+\frac{E^{(1)} L}{\pi} \frac{\chi}{\Omega}\left(\frac{h^{2}}{8}+\frac{\alpha h^{2}}{2}\right) \cos \left(\frac{\pi x}{L}\right)-\frac{E^{(2)} L}{\pi} \frac{\chi}{\Omega}\left(\frac{z^{2}}{2}-\alpha h z\right) \cos \left(\frac{\pi x}{L}\right) \\
-\frac{E^{(1)} h \pi}{L D_{3} \Omega}\left[-\left(\frac{h C_{1}}{2}\right)+C_{2} \frac{h(1+2 \alpha)}{\pi} \cos \left(\frac{\pi}{2} \frac{-\alpha}{0.5+\alpha}\right)\right] \cos \left(\frac{\pi x}{L}\right) \\
+\frac{E^{(2)} h \pi}{L D_{3} \Omega}\left[z C_{3}+\frac{h(1-2 \alpha)}{\pi} \cos \left(\frac{\pi}{2} \frac{(z / h-\alpha)}{0.5-\alpha}\right)+\frac{h(1-2 \alpha)}{\pi} \cos \left(\frac{\pi}{2} \frac{-\alpha}{0.5-\alpha}\right)\right] \cos \left(\frac{\pi x}{L}\right)
\end{array}\right\}
$$

The results of simply supported beam subjected to sinusoidal load, for maximum non-dimensional transverse displacement, axial or normal bending stresses and transverse shear stresses are presented in Table 1 and Table 2 and graphically presented in Fig.2 through 10. 
Table 1 Non-dimensional maximum transverse displacement $(\bar{w})$ at $(x=0.5 L, z=0)$, axial displacement $(\bar{u})$ at $(x=0, z= \pm h / 2)$, axial stress $\left(\bar{\sigma}_{x}\right)$ at $(x=0.5 L, z= \pm h / 2)$, and transverse shear stresses $\left(\bar{\tau}_{x z}\right)$ at $(x=0.0, z=a h)$ for simply supported beam subjected to sinusoidal load $q=q_{0} \sin (\pi x / L)$ with aspect ratio, $S=4$ (Example: 1 )

\begin{tabular}{|c|c|c|c|c|c|c|c|c|c|}
\hline \multirow{2}{*}{$\begin{array}{l}\text { Source } \\
\text { (Model) }\end{array}$} & \multirow{2}{*}{$\bar{w}$} & \multicolumn{3}{|c|}{$\bar{u}$} & \multicolumn{3}{|c|}{$\bar{\sigma}_{x}$} & \multirow{2}{*}{$\bar{\tau}_{x z}^{C R}$} & \multirow{2}{*}{$\bar{\tau}_{x z}^{E E}$} \\
\hline & & $-h / 2$ & & $h / 2$ & $-h / 2$ & & $h / 2$ & & \\
\hline Present (LTSDT) & 4.7437 & 5.0353 & - & 1.5574 & 3.9547 & - & 30.5650 & 2.9895 & 2.6784 \\
\hline Bernoulli- Euler (ETB) & 2.6281 & 3.8574 & - & 1.4216 & 3.0386 & - & 27.9540 & -- & 2.9453 \\
\hline Timoshenko [1] (FSDT) & 4.7967 & 3.8574 & - & 1.4216 & 3.0386 & - & 27.9540 & 1.8189 & 2.9453 \\
\hline Ghugal and Shimpi [22], (LTSDT-II) & 4.7437 & 5.0353 & & --- & 3.9547 & - & 30.5650 & 2.9895 & 2.6784 \\
\hline Kant and Manjunatha [5] (FSDT/FEM) & 4.2828 & 4.8611 & & --- & 3.7490 & - & 27.0500 & 1.9270 & 2.8240 \\
\hline Lu and Liu [20] (HSDT) & 4.7773 & 4.7143 & & --- & 3.5714 & - & 30.0000 & 2.7925 & -- \\
\hline Maiti and Sinha [9] (HST/FEM) & 3.5346 & --- & & --- & 2.3599 & - & 25.7834 & --- & 2.4252 \\
\hline Maiti and Sinha [9] (FSDT/ FEM) & 4.7898 & --- & & --- & 3.1514 & - & 29.1144 & 2.8467 & --- \\
\hline Vinayak et al. [10] (HSDT/ FEM) & 4.5619 & --- & & --- & 4.0000 & - & 27.0000 & --- & 2.7500 \\
\hline Catapano et al [21] for $N=3$ & 4.5733 & 4.4228 & & --- & 3.7491 & - & 28.757 & $\begin{array}{c}2.1854 \\
(0.25 L)\end{array}$ & --- \\
\hline Ghugal [24] (Exact Solution) & 4.6616 & 4.5685 & - & 1.5288 & 3.8381 & - & 30.0190 & 2.7212 & --- \\
\hline
\end{tabular}

Figure in parenthesis indicate the distance $x$ from left end of the beam.

Table 2 Nondimensional maximum transverse displacement $(\bar{w})$ at $(x=0.5 L, z=0)$ axial displacement $(\bar{u})$ at $(x=0, z= \pm h / 2)$, axial stress $\left(\bar{\sigma}_{x}\right)$ at $(x=0.5 L, z= \pm h / 2)$, and transverse shear stresses $\left(\bar{\tau}_{x z}\right)$ at $(x=0.0, z=a h)$ for simply supported beam subjected to sinusoidal load $q=q_{0} \sin (\pi x / L)$ with aspect ratio, $S=10$ (Example: 1 )

\begin{tabular}{|c|c|c|c|c|c|c|c|c|c|}
\hline \multirow{2}{*}{$\begin{array}{l}\text { Source } \\
\text { (Model) }\end{array}$} & \multirow{2}{*}{$\bar{w}$} & \multicolumn{3}{|c|}{$\bar{u}$} & \multicolumn{3}{|c|}{$\bar{S}_{x}$} & \multirow{2}{*}{$\epsilon_{x z}^{C R}$} & \multirow{2}{*}{$\epsilon_{x z}^{E E}$} \\
\hline & & $-h / 2$ & & $h / 2$ & $-h / 2$ & & $h / 2$ & & \\
\hline Present (TSDT) & 2.9744 & 63.305 & - & 22.563 & 19.888 & - & 177.14 & 7.6948 & 7.2609 \\
\hline Bernoulli- Euler (ETB) & 2.6281 & 60.354 & - & 22.213 & 18.935 & - & 174.71 & -- & 7.3643 \\
\hline Timoshenko [1] (FSDT) & 2.9730 & 60.354 & - & 22.213 & 18.935 & - & 174.71 & 4.5473 & 7.3643 \\
\hline Ghugal and Shimpi [22] (LTSDT-II) & 2.9744 & 63.305 & & -- & 19.888 & - & 174.71 & 7.6948 & 7.2638 \\
\hline $\begin{array}{l}\text { Manjunatha and Kant } \\
\text { [8] (HOSTB5/ FEM) }\end{array}$ & 2.8986 & -- & & -- & 19.710 & - & 173.00 & 4.9130 & 7.2820 \\
\hline $\begin{array}{l}\text { Lu and Liu }[20] \\
(\text { HSDT) }\end{array}$ & 3.0000 & 61.666 & & -- & 20.000 & - & 175.00 & 7.3000 & -- \\
\hline $\begin{array}{l}\text { Catapano et al [ 21 ] } \\
\text { for } N=3\end{array}$ & 2.9332 & 61.637 & & --- & 19.693 & & 175.31 & $\begin{array}{r}5.5649 \\
(0.25 L)\end{array}$ & \\
\hline $\begin{array}{l}\text { Ghugal[24] } \\
\text { (Exact Solution) }\end{array}$ & 2.9613 & 62.326 & - & 22.476 & 19.830 & - & 176.53 & 7.2678 & --- \\
\hline
\end{tabular}

Figure in parenthesis indicate the distance $x$ from left end of the beam. 


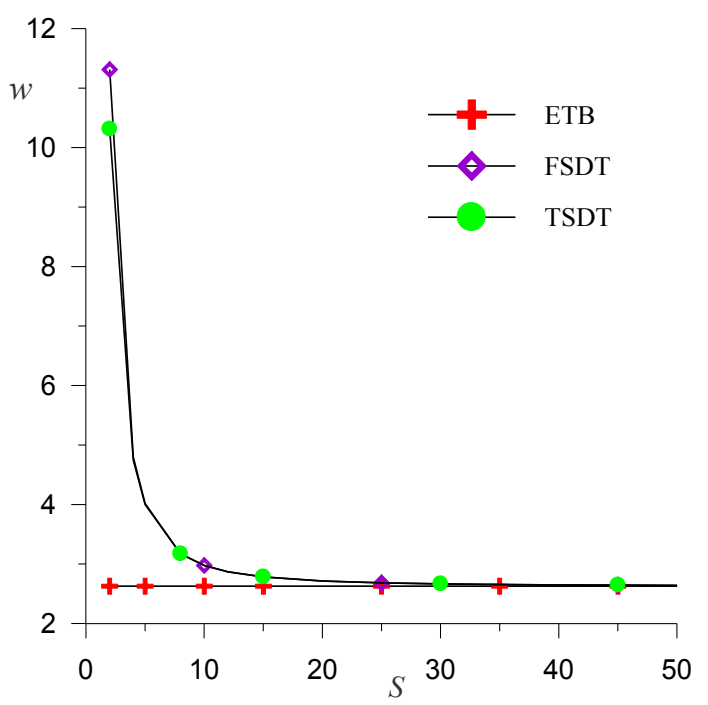

Figure 2 Variation of maximum transverse displacement $(\bar{w})$ of simply supported beam at $(x=L / 2, S)$ when subjected to sinusoidal load

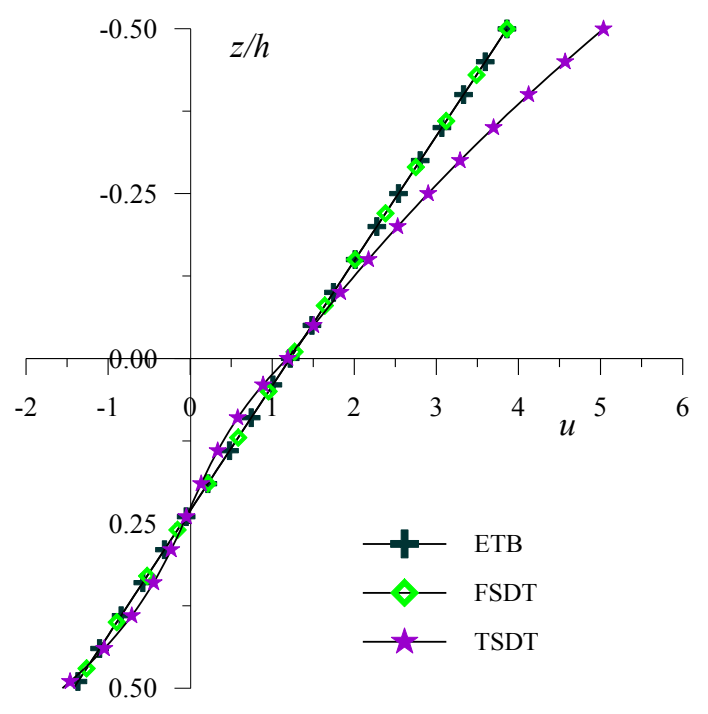

Figure 3 Variation of axial displacement $(\bar{u})$ through the thickness of simply supported beam at $(x=0, z)$ when subjected to sinusoidal load for aspect ratio 4 


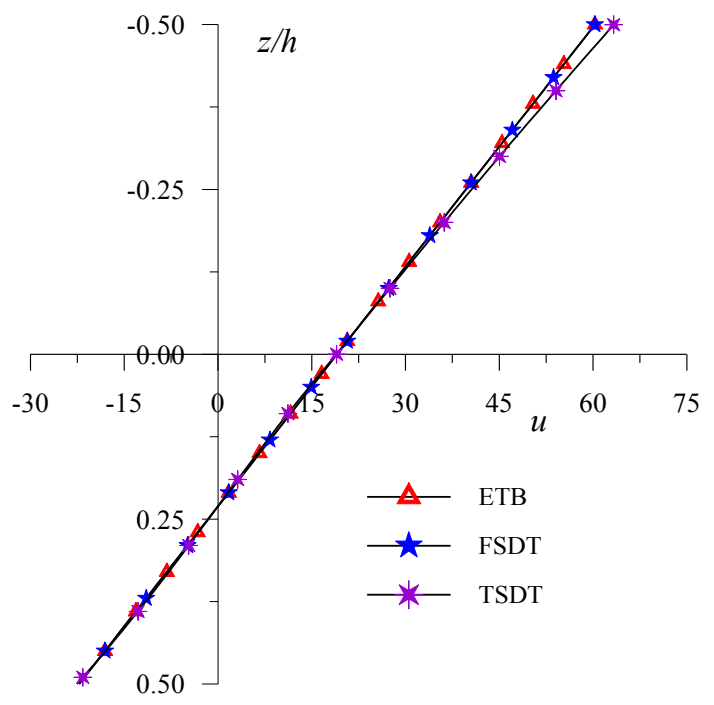

Figure 4 Variation of axial displacement $(\bar{u})$ through the thickness of simply supported beam at $(x=0, z)$ when subjected to sinusoidal load for aspect ratio 10

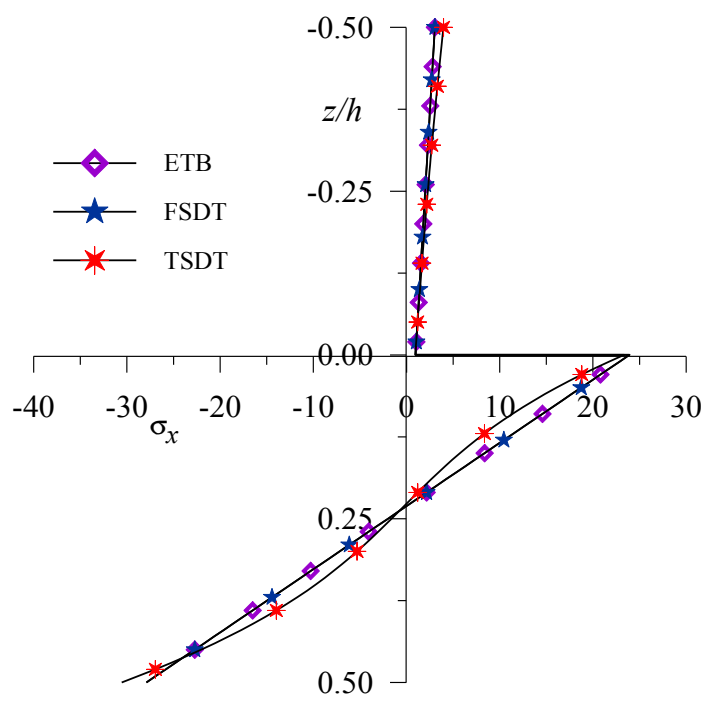

Figure 5 Variation of axial stress $\left(\bar{\sigma}_{x}\right)$ through the thickness of simply supported beam at $(x=L / 2, z)$ when subjected to sinusoidal load for aspect ratio 4 


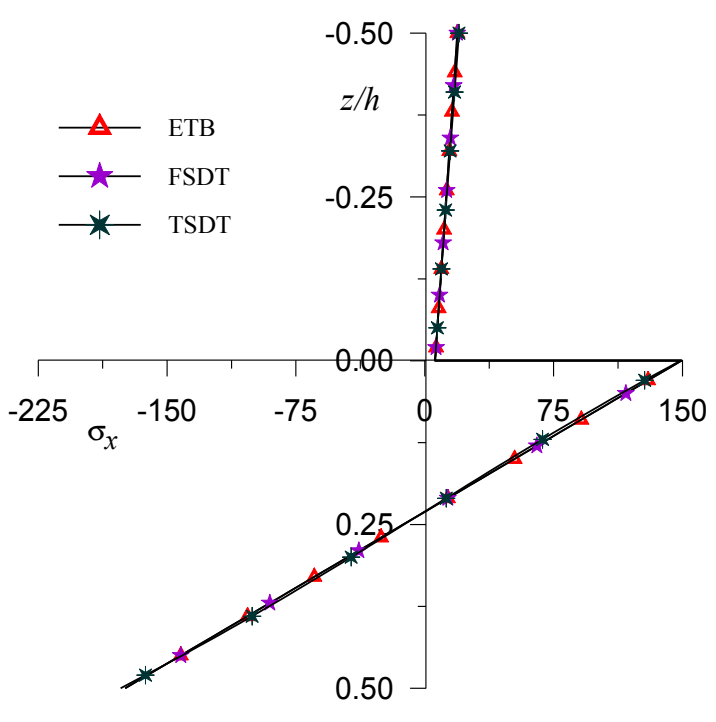

Figure 6 Variation of Axial stress $\left(\bar{\sigma}_{x}\right)$ through the thickness of simply supported beam at $(x=L / 2, z)$ when subjected to sinusoidal load for aspect ratio 10

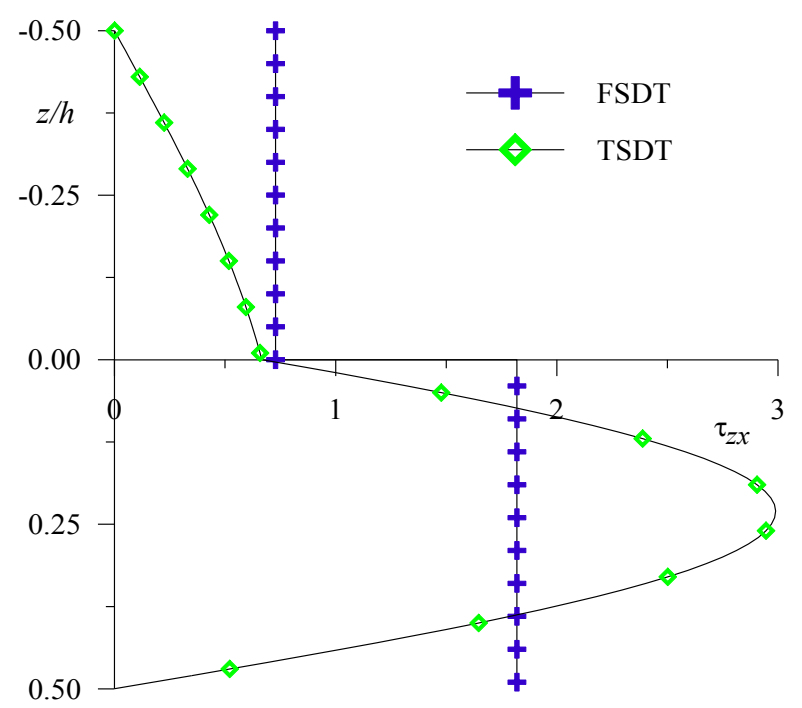

Figure 7 Variation of transverse shear stress $\left(\bar{\tau}_{z x}\right)$ through the thickness of simply supported beam at $(x=0.0 L, z)$ when subjected to sinusoidal load and obtained using constitutive relations for aspect ratio 4 


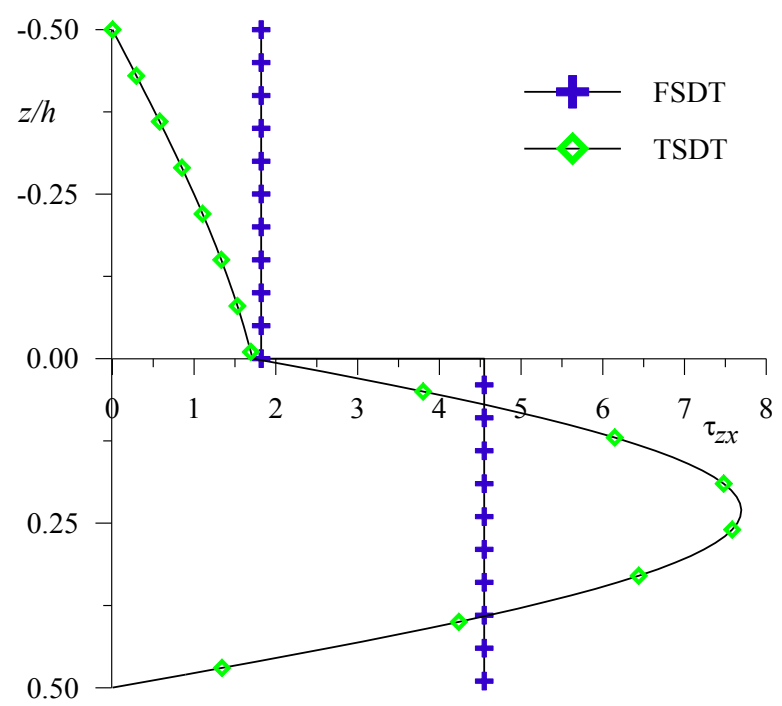

Figure 8 Variation of transverse shear stress $\left(\bar{\tau}_{z x}\right)$ through the thickness of simply supported beam at $(x=0.0 L, z)$ when subjected to sinusoidal load and obtained using constitutive relations for aspect ratio 10

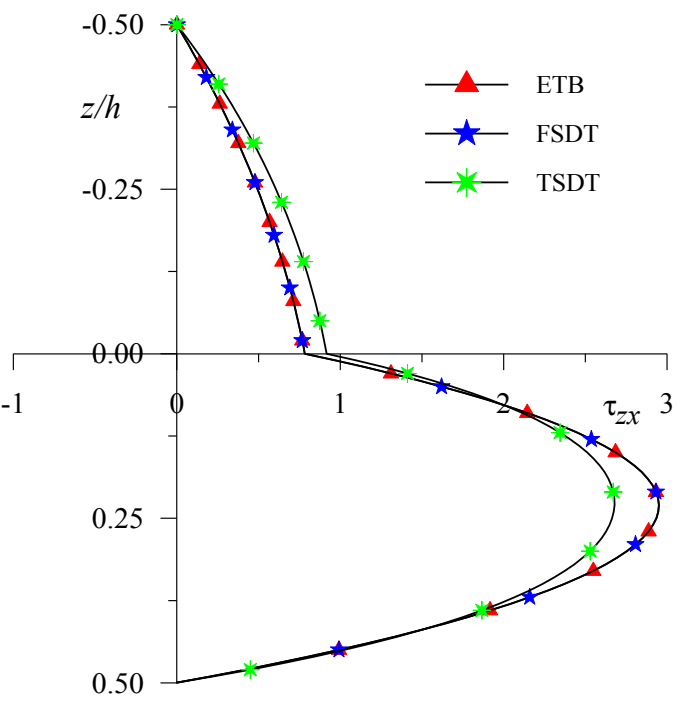

Figure 9 Variation of transverse shear stress $\left(\bar{\tau}_{z x}\right)$ through the thickness of simply supported beam at $(x=0, z)$ when subjected to sinusoidal load and obtained using equilibrium equations for aspect ratio 4 


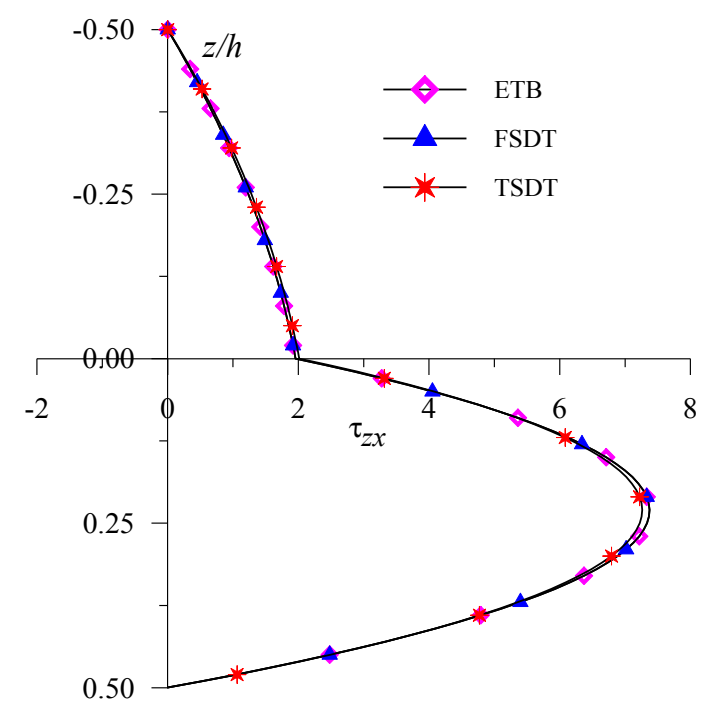

Figure 10 Variation of transverse shear stress $\left(\bar{\tau}_{z x}\right)$ through the thickness of simply supported beam at $(x=0, z)$ when subjected to sinusoidal load and obtained using equilibrium equations for aspect ratio 10

\subsection{Example 2: Fixed beam with sinusoidal load $q=q_{0} \sin (\pi x / L)$}

A fixed-fixed beam with rectangular cross-section $(b \times h)$ is considered. The beam is subjected to sinusoidal load over the span $L$ at surface $z=-h / 2$. The origin of the beam is at left end support, i.e. at $x=0$. The boundary conditions associated with fixed beam are as follows:

$$
\begin{gathered}
D \frac{d w}{d x}=D w=0 \quad \text { at } x=0 \\
D \frac{d^{3} w}{d x^{3}}=D \frac{d^{2} \phi}{d x^{2}}=D \frac{d w}{d x}=0 \text { at } x=\frac{L}{2} \\
\phi=0 \text { at } x=0, \frac{L}{2}
\end{gathered}
$$

Thus, the general solution for $\phi$ and $w$ are obtained as follows:

$$
\phi(x)=\xi(\sinh \lambda x-\cosh \lambda x+\cos \pi x / L) \quad \text { when } \quad 0 \leq x \leq L / 2
$$

where $\xi=\frac{q_{0} L}{\pi D D_{3} \Omega}$ 


$$
w(x)=\frac{q_{0} L^{4}}{\pi^{4} D} \frac{\chi}{\Omega}\left[\sin (\pi x / L)-(\pi x / L)+\left(\pi x^{2} / L^{2}\right)\right]+\frac{q_{0} L^{2}}{\pi D} \frac{D_{1}}{D_{3} \Omega}\left[\frac{\cosh \lambda x-\sinh \lambda x-1}{\lambda L}+\frac{x}{L}-\frac{x^{2}}{L^{2}}\right]
$$

when $0 \leq x \leq L / 2 \quad$ where $\Omega=\left(1+\frac{\pi^{2}}{\lambda^{2} L^{2}}\right), \chi=\left(1+\frac{D_{2}}{D_{3}} \frac{\pi^{2}}{L^{2}}\right)$,

The expressions for displacement and stresses of the beam are obtained using this solution, are as follows:

The axial displacement for Layer 1 is expressed as:

$$
u^{(1)}=\frac{q_{0}}{D}\left\{\begin{array}{l}
-\frac{(z-\alpha h) L^{3}}{\pi^{3}} \frac{\chi}{\Omega}[\cos (\pi x / L)-1+2(x / L)] \\
-\frac{(z-\alpha h) L}{\pi \Omega} \frac{D_{1}}{D_{3}}[\sinh \lambda x-\cosh \lambda x+1-2(x / L)] \\
+\frac{L h}{\pi D_{3} \Omega}\left[C_{1}+C_{2} \sin \left(\frac{\pi}{2} \frac{z / h-\alpha}{0.5+\alpha}\right)\right] f_{1}(x)
\end{array}\right\}
$$

The axial displacement for Layer 2 is expressed as:

$$
u^{(2)}=\frac{q_{0}}{D}\left\{\begin{array}{l}
-\frac{(z-\alpha h) L^{3}}{\pi^{3}} \frac{\chi}{\Omega}[\cos (\pi x / L)-1+2(x / L)] \\
-\frac{(z-\alpha h) L}{\pi \Omega} \frac{D_{1}}{D_{3}}[\sinh \lambda x-\cosh \lambda x+1-2(x / L)] \\
+\frac{L h}{\pi D_{3} \Omega}\left[C_{3}+\sin \left(\frac{\pi}{2} \frac{z / h-\alpha}{0.5-\alpha}\right)\right] f_{1}(x)
\end{array}\right\}
$$

where $f_{1}(x)=\sinh \lambda x-\cosh \lambda x+\cos (\pi x / L)$

The axial stress for Layer 1 is expressed as:

$$
\sigma_{x}^{(1)}=\frac{q_{0}}{D}\left\{\begin{array}{l}
-\frac{E^{(1)}(z-\alpha h) L^{3}}{\pi^{3}} \frac{\chi}{\Omega}\left[\frac{\pi}{L} \sin (\pi x / L)+(2 / L)\right] \\
-\frac{E^{(1)}(z-\alpha h) L}{\pi \Omega} \frac{D_{1}}{D_{3}}(\lambda \cosh \lambda x-\lambda \sinh \lambda x-2 / L) \\
+\frac{E^{(1)} \lambda L h}{\pi D_{3} \Omega}\left[C_{1}+C_{2} \sin \left(\frac{\pi}{2} \frac{z / h-\alpha}{0.5+\alpha}\right)\right] f_{2}(x)
\end{array}\right\}
$$


where $f_{2}(x)=\cosh \lambda x-\sinh \lambda x-\frac{\pi}{\lambda L} \sin (\pi x / L)$

The axial stress for Layer 2 is expressed as:

$$
\sigma_{x}^{(2)}=\frac{q_{0}}{D}\left\{\begin{array}{l}
-\frac{E^{(2)}(z-\alpha h) L^{3}}{\pi^{3}} \frac{\chi}{\Omega}\left[\frac{\pi}{L} \sin (\pi x / L)+(2 / L)\right] \\
-\frac{E^{(2)}(z-\alpha h) \lambda L}{\pi \Omega} \frac{D_{1}}{D_{3}}\left(\cosh \lambda x-\sinh \lambda x-\frac{2}{\lambda L}\right) \\
+\frac{E^{(2)} \lambda L h}{\pi D_{3} \Omega}\left[C_{3}+\sin \left(\frac{\pi}{2} \frac{z / h-\alpha}{0.5-\alpha}\right)\right] f_{2}(x)
\end{array}\right\}
$$

The transverse shear stress using constitutive relationship for Layer 1 is expressed as:

$$
\begin{aligned}
\tau_{z x}^{(1)} & =\frac{G^{(1)} \xi \pi C_{2}}{(1+2 \alpha)} \cos \left(\frac{\pi}{2} \frac{z / h-\alpha}{0.5+\alpha}\right) f_{1}(x) \\
\tau_{z x}^{(2)} & =\frac{G^{(2)} \xi \pi}{(1-2 \alpha)} \cos \left(\frac{\pi}{2} \frac{z / h-\alpha}{0.5-\alpha}\right) f_{1}(x)
\end{aligned}
$$

The transverse shear stress using equilibrium equations for Layer 1 is expressed as:

$$
\tau_{z x}^{(1)}=\frac{q_{0}}{D}\left\{\begin{array}{l}
-\frac{E^{(1)} L}{\pi} \frac{\chi}{\Omega}\left(\frac{z^{2}}{2}-\alpha h z-\frac{h^{2}}{8}-\frac{\alpha h^{2}}{2}\right) \cos (\pi x / L) \\
+\frac{E^{(1)} \lambda^{2} L^{2}}{\pi L \Omega} \frac{D_{1}}{D_{3}}\left(\frac{z^{2}}{2}-\alpha h z-\frac{h^{2}}{8}-\frac{\alpha h^{2}}{2}\right)[\sinh \lambda x-\cosh \lambda x] \\
-\frac{E^{(1)} \lambda^{2} L^{2} h}{\pi \Omega} \frac{D_{1}}{D_{3}}\left(z C_{1}-\frac{h(1+2 \alpha)}{\pi} \cos \left(\frac{\pi}{2} \frac{z / h-\alpha}{0.5+\alpha}\right)+\frac{h C_{1}}{2}\right) \\
{\left[\sinh \lambda x-\cosh \lambda x-\frac{\pi^{2}}{\lambda^{2} L^{2}} \cos \frac{\pi x}{L}\right]}
\end{array}\right\}
$$

The transverse shear stress using equilibrium equations for Layer 2 is expressed as: 


$$
\tau_{z x}^{(2)}=\frac{q_{0}}{D}\left\{\begin{array}{l}
-\frac{E^{(2)} L}{\pi} \frac{\chi}{\Omega}\left(\frac{z^{2}}{2}-\alpha h z\right) \cos \frac{\pi x}{L}+\frac{E^{(1)} L}{\pi} \frac{\chi}{\Omega}\left(\frac{h^{2}}{8}+\frac{\alpha h^{2}}{2}\right) \cos \frac{\pi x}{L} \\
+\frac{E^{(2)} \lambda^{2} L^{2}}{\pi L \Omega} \frac{D_{1}}{D_{3}}\left(\frac{z^{2}}{2}-\alpha h z\right)[\sinh \lambda x-\cosh \lambda x] \\
-\frac{E^{(1)} \lambda^{2} L^{2}}{\pi L \Omega} \frac{D_{1}}{D_{3}}\left(\frac{h^{2}}{8}+\frac{\alpha h^{2}}{2}\right)[\sinh \lambda x-\cosh \lambda x] \\
-\frac{E^{(2)} \lambda^{2} L^{2} h}{\pi D_{3} \Omega L}\left[z C_{3}-\frac{h(1-2 \alpha)}{\pi} \cos \left(\frac{\pi}{2} \frac{z / h-\alpha}{0.5-\alpha}\right)+\frac{h(1-2 \alpha)}{\pi} \cos \left(\frac{\pi}{2} \frac{-\alpha}{0.5-\alpha}\right)\right] \\
{\left[\begin{array}{l}
\left.\sinh \lambda x-\cosh \lambda x-\frac{\pi^{2}}{\lambda^{2} L^{2}} \cos \frac{\pi x}{L}\right] \\
-\frac{E^{(1)} \lambda^{2} L^{2} h}{\pi D_{3} \Omega L}\left[-\frac{h(1+2 \alpha)}{\pi} \cos \left(\frac{\pi}{2} \frac{-\alpha}{0.5+\alpha}\right)+\frac{h C_{1}}{2}\right]\left[\sinh \lambda x-\cosh \lambda x-\frac{\pi^{2}}{\lambda^{2} L^{2}} \cos \frac{\pi x}{L}\right.
\end{array}\right]}
\end{array}\right\}
$$

The results of fixed beam in Example 2 subjected to sinusoidal load, for maximum nondimensional transverse displacement, axial or normal bending stress and transverse shear stress are presented in Table 3 and graphically presented in Figs.11 through 20. The results of axial stresses are presented at $x=0$ and $x=\alpha_{0} L$ from left end support.

Table 3 Non-dimensional maximum transverse displacement $(\bar{w})$ at $(x=L / 2, z=0.0)$, axial displacement $(\bar{u})$ at $(x=0.25 L$, $z= \pm h / 2)$, axial stress $\left(\bar{\sigma}_{x}\right)$ at $(x=0.0 L, z= \pm h / 2)$, and transverse shear stresses $\left(\bar{\tau}_{x z}\right)$ at $(x=0.0, z=a h)$ for fixed beam subjected to sinusoidal load $q=q_{0} \sin (\pi x / L)$ with aspect ratio, $S=4,10$ (Example: 2 )

\begin{tabular}{|c|c|c|c|c|c|c|c|c|}
\hline \multirow{2}{*}{$\begin{array}{l}\text { Source } \\
\text { (Model) }\end{array}$} & \multirow{2}{*}{$S$} & \multirow{2}{*}{$\bar{w}$} & \multicolumn{2}{|c|}{$\bar{u}$} & \multicolumn{2}{|c|}{$\bar{\sigma}_{x}$} & \multirow{2}{*}{$\bar{\tau}_{x z}^{C R}$} & \multirow{2}{*}{$\bar{\tau}_{x z}^{E E}$} \\
\hline & & & $-h / 2$ & $h / 2$ & $-h / 2$ & $h / 2$ & & \\
\hline $\begin{array}{l}\text { Present } \\
\text { (TSDT) }\end{array}$ & & 1.9361 & $\begin{array}{c}2.6059 \\
(0.25 L)\end{array}$ & $\begin{array}{c}-0.69562 \\
(0.25 L)\end{array}$ & & & $\begin{array}{l}2.2796 \\
(0.1 L)\end{array}$ & $\begin{array}{c}-4.9815 \\
(0.00)\end{array}$ \\
\hline $\begin{array}{l}\text { Present } \\
(\mathrm{TSDT})\end{array}$ & & -- & $\begin{array}{c}1.5602 \\
(0.3758 L)\end{array}$ & $\begin{array}{l}-0.43169 \\
(0.3758 L)\end{array}$ & $\begin{array}{c}3.0137 \\
(0.0745 L)\end{array}$ & $\begin{array}{c}-38.279 \\
(0.0745 L)\end{array}$ & $\begin{array}{l}2.3120 \\
(0.20 L)\end{array}$ & $\begin{array}{l}1.8942 \\
(0.20 L)\end{array}$ \\
\hline $\begin{array}{l}\text { Bernoulli- Euler } \\
\text { (ETB) }\end{array}$ & 4 & 0.5634 & $\begin{array}{c}1.5602 \\
(0.25 L)\end{array}$ & $\begin{array}{c}-0.57502 \\
(0.25 L)\end{array}$ & $\begin{array}{l}3.0137 \\
(0.00)\end{array}$ & $\begin{array}{c}-27.757 \\
(0.00)\end{array}$ & -- & $\begin{array}{c}2.9503 \\
(0.00)\end{array}$ \\
\hline $\begin{array}{l}\text { Timoshenko [1] } \\
\text { (FSDT) }\end{array}$ & 4 & 1.9530 & $\begin{array}{l}1.5602 \\
(0.25 L)\end{array}$ & $\begin{array}{c}-0.57506 \\
(0.25 L) \\
\end{array}$ & $\begin{array}{r}3.0137 \\
(0.00) \\
\end{array}$ & $\begin{array}{c}-27.757 \\
(0.00) \\
\end{array}$ & $\begin{array}{c}2.2736 \\
(0.00) \\
\end{array}$ & $\begin{array}{c}2.9503 \\
(0.00) \\
\end{array}$ \\
\hline $\begin{array}{l}\text { Present } \\
(\mathrm{TSDT})\end{array}$ & & 0.9124 & $\begin{array}{c}14.625 \\
(0.25 L)\end{array}$ & $\begin{array}{l}-4.8476 \\
(0.25 L)\end{array}$ & $24.706(0.00)$ & - $440.59(0.00)$ & $\begin{array}{l}7.1983 \\
(0.1 L)\end{array}$ & $\begin{array}{c}-12.532 \\
(0.00)\end{array}$ \\
\hline $\begin{array}{l}\text { Present } \\
(\mathrm{TSDT})\end{array}$ & & -- & $\begin{array}{c}12.482 \\
(0.3143 L)\end{array}$ & $\begin{array}{c}-4.1818 \\
(0.3143 L)\end{array}$ & $\begin{array}{c}12.0547 \\
(0.03937 L)\end{array}$ & $\begin{array}{c}-141.01 \\
(0.03937 L)\end{array}$ & $\begin{array}{c}6.2222 \\
(0.20 L)\end{array}$ & $\begin{array}{l}5.1749 \\
(0.20 L)\end{array}$ \\
\hline $\begin{array}{l}\text { Bernoulli- Euler } \\
\text { (ETB) }\end{array}$ & 1 & 0.5634 & $\begin{array}{c}12.482 \\
(0.25 L)\end{array}$ & $\begin{array}{c}-4.6002 \\
(0.25 L)\end{array}$ & $12.0547(0.00)$ & $\begin{array}{c}-111.03 \\
(0.00)\end{array}$ & -- & $\begin{array}{l}7.3757 \\
(0.00)\end{array}$ \\
\hline $\begin{array}{l}\text { Timoshenko [1] } \\
\text { (FSDT) }\end{array}$ & 0 & 0.9108 & $\begin{array}{c}12.482 \\
(0.25 L)\end{array}$ & $\begin{array}{c}-4.6005 \\
(0.25 L) \\
\end{array}$ & $\begin{array}{c}12.0547 \\
(0.00) \\
\end{array}$ & $\begin{array}{c}111.03 \\
(0.00) \\
\end{array}$ & $\begin{array}{l}4.5473 \\
(0.00) \\
\end{array}$ & $\begin{array}{l}7.3757 \\
(0.00) \\
\end{array}$ \\
\hline
\end{tabular}

Figures in parenthesis indicate the distance $x$ from left end of the beam. 


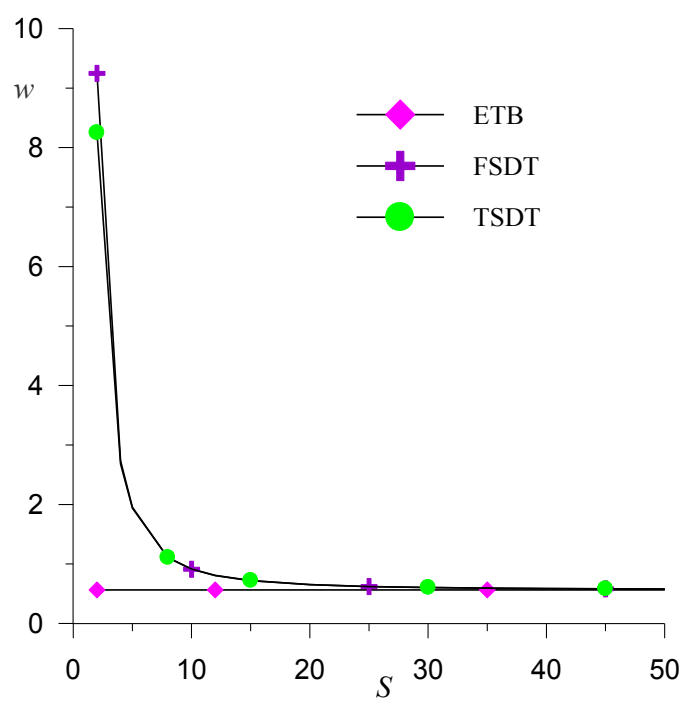

Figure 11 Variation of maximum transverse displacement $(\bar{w})$ of fixed beam at $(x=L / 2, S)$ when subjected to sinusoidal load

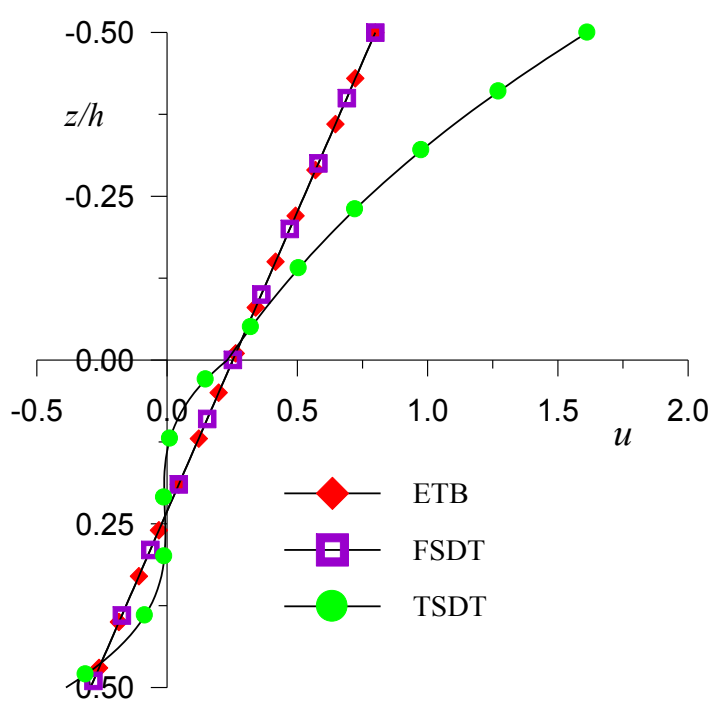

Figure 12 Variation of axial displacement $(u)$ through the thickness of fixed beam at $(x=0.25 L, z)$ when subjected to sinusoidal load for aspect ratio 4 


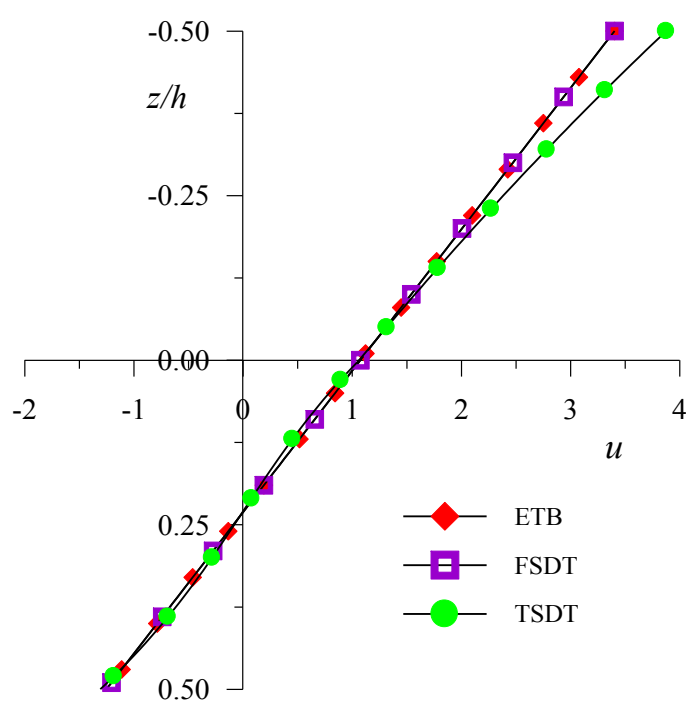

Figure 13 Variation of axial displacement $(u)$ through the thickness of fixed beam at $(x=0.25 L, z)$ when subjected to sinusoidal load for aspect ratio 10

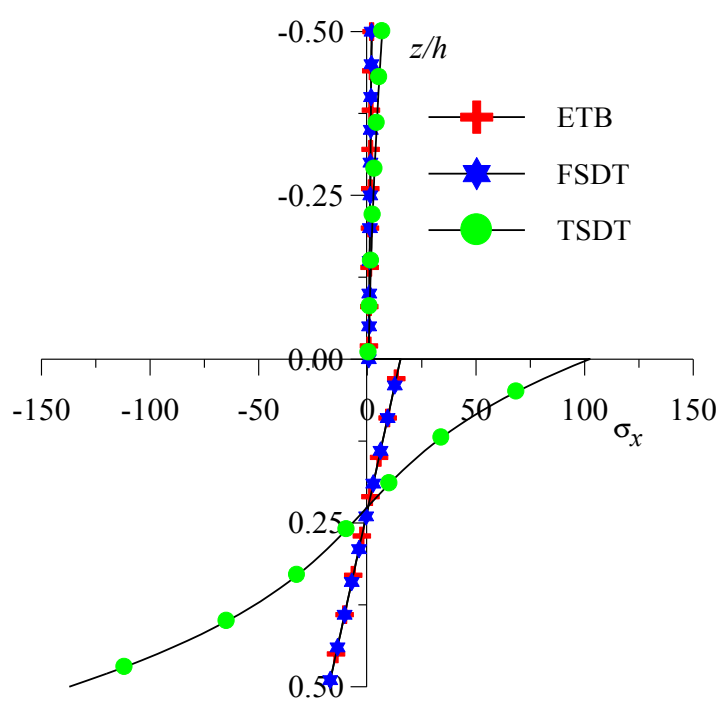

Figure 14 Variation of Axial stress $\left(\bar{\sigma}_{x}\right)$ through the thickness of fixed beam at $(x=0, z)$ when subjected to sinusoidal load for aspect ratio 4 


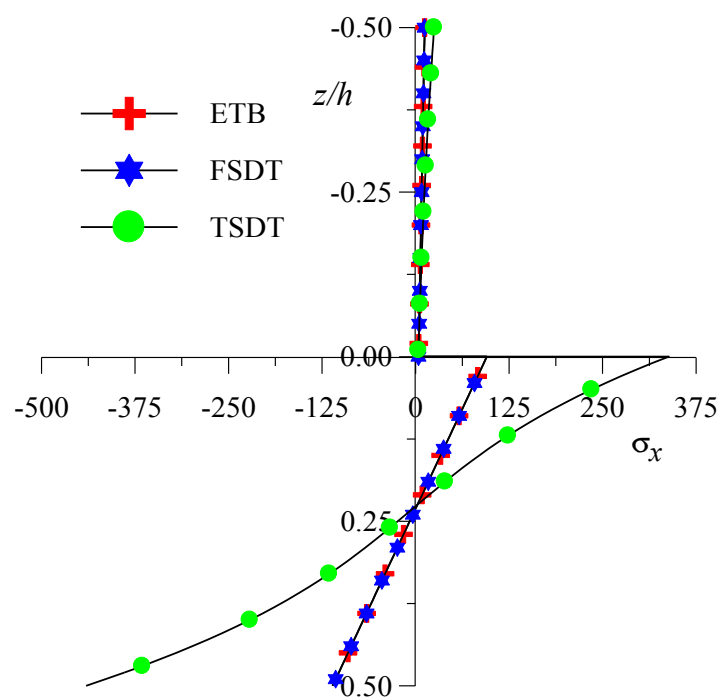

Figure 15 Variation of axial stress $\left(\bar{\sigma}_{x}\right)$ through the thickness of fixed beam at $(x=0, z)$ when subjected to sinusoidal load for aspect ratio 10

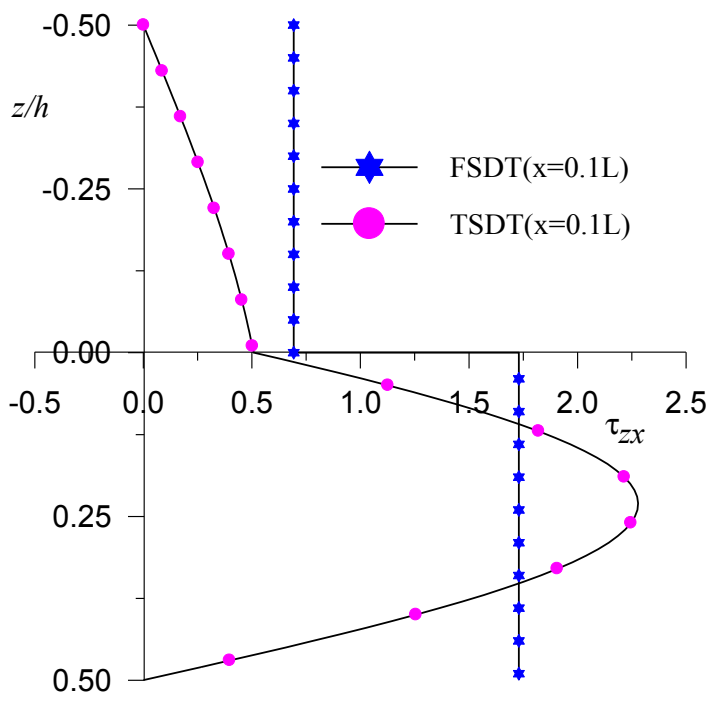

Figure 16 Variation of transverse shear stress $\left(\bar{\tau}_{z x}\right)$ through the thickness of fixed beam at $(x=0.1 L, z)$ when subjected to sinusoidal load and obtained using constitutive relations for aspect ratio 4 


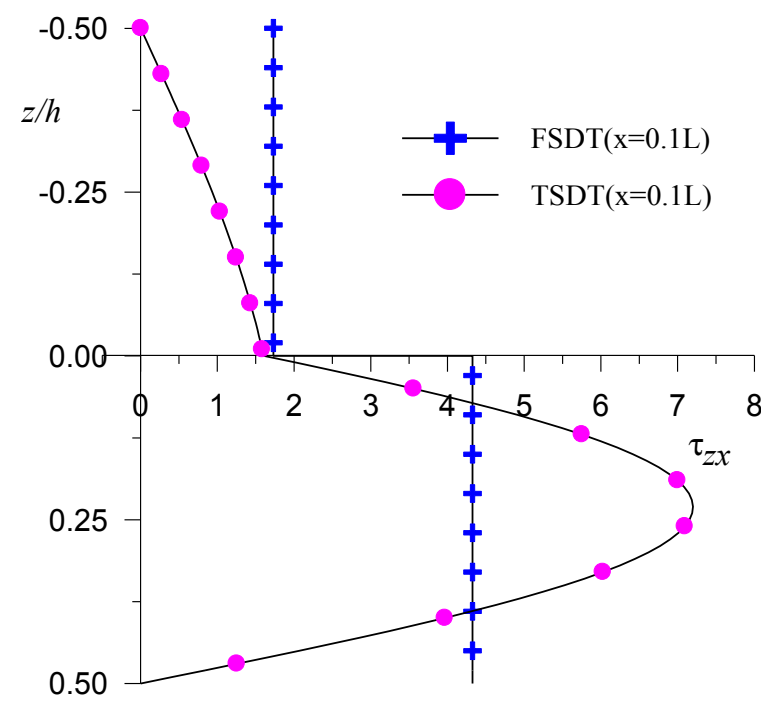

Figure 17 Variation of transverse shear stress $\left(\bar{\tau}_{z x}\right)$ through the thickness of fixed beam at $(x=0.1 L, z)$ when subjected to sinusoidal load and obtained using costitutive relations for aspect ratio 10

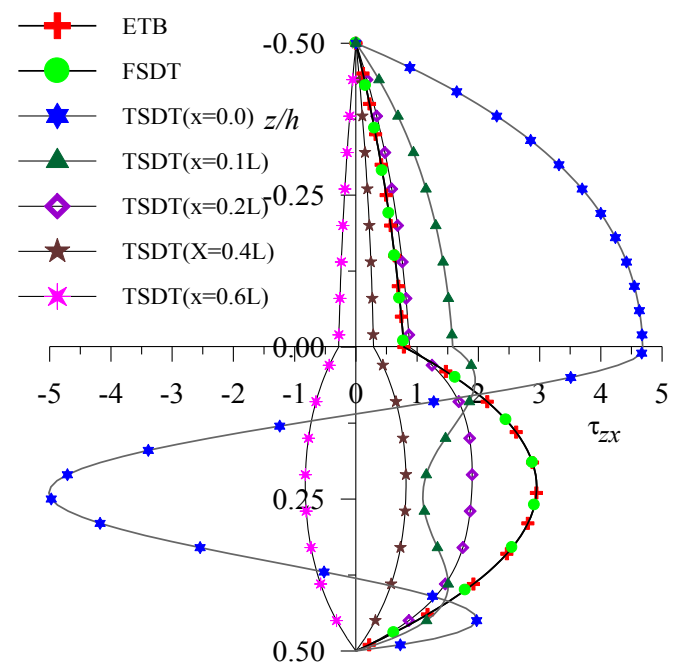

Figure 18 Variation of transverse shear stress $\left(\bar{\tau}_{z x}\right)$ through the thickness of fixed beam at $\left(x=\alpha_{0} L, z\right)$ when subjected to sinusoidal load and obtained using equilibrium equations for aspect ratio 4 


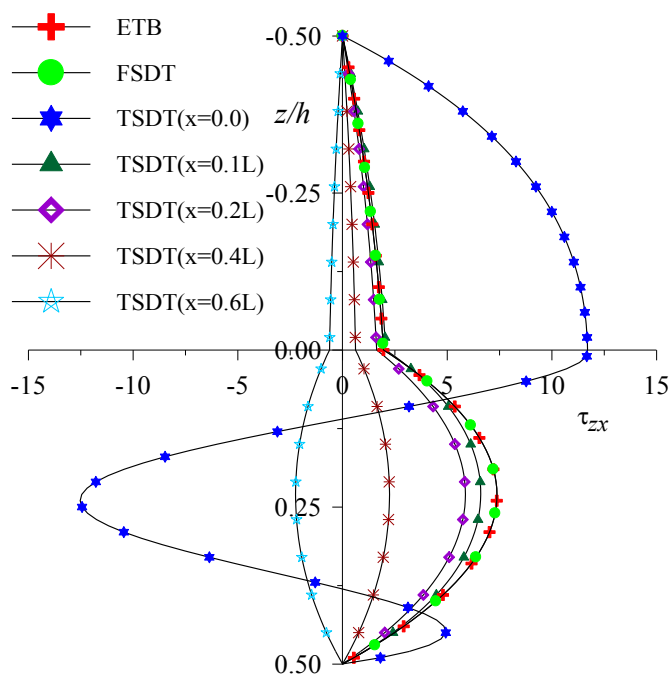

Figure 19 Variation of transverse shear stress $\left(\bar{\tau}_{z x}\right)$ through the thickness of fixed beam at $\left(x=\alpha_{0} L, z\right)$ when subjected to sinusoidal load and obtained using equilibrium equations for aspect ratio 10

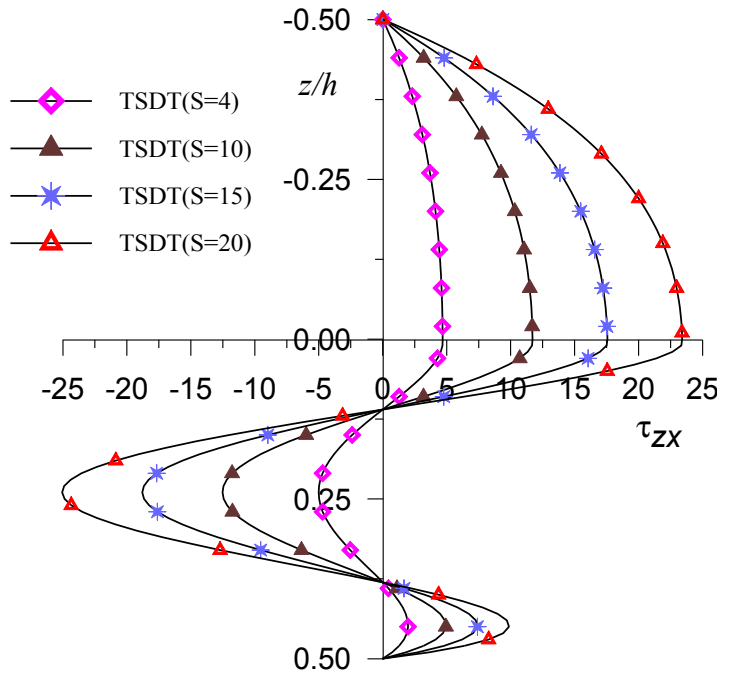

Figure 20 Variation of transverse shear stress $\left(\bar{\tau}_{z x}\right)$ through the thickness of fixed beam at $(x=0, z)$ when subjected to sinusoidal load and obtained using equilibrium equations for aspect ratios $(S=4,10,15,20)$

The percentage error in results obtained by models of other researchers with respect to the corresponding results obtained by present theory is calculated as follows:

Latin American Journal of Solids and Structures 10(2013) $675-705$ 


$$
\% \text { error }=\frac{\text { value by a particular model }- \text { value by present theory }}{\text { value by present theory }} \times 100
$$

\section{DISCUSSION OF RESULTS}

The results for axial displacement, transverse displacement, axial stresses and transverse stresses in this paper are presented in the following non-dimensional form for the purpose of comparison:

$$
\bar{u}=\frac{E^{(1)} b u}{q h} ; \quad \bar{w}=\frac{100 E^{(1)} b h^{3} w}{q L^{4}} ; \quad \bar{\sigma}_{x x}=\frac{b \sigma_{x x}}{q} ; \quad \bar{\tau}_{x z}=\frac{b \tau_{x z}}{q}
$$

The results obtained by present theory (TSDT) for displacement and stresses are compared with the ETB, FSDT of Timoshenko, Kant and Manjunatha, Maiti and Sinha, and Vinayak et al., LTSDT of Shimpi and Ghugal [22] and exact elasticity solution [24] wherever applicable for composite laminated beam subjected to single sinusoidal load. The exact solution for fixed crossply laminated beam subjected to sinusoidal load is not available; hence the results are compared with ETB and FSDT.

\subsection{Transverse displacement $(\bar{w})$ :}

The results of maximum non-dimensionalised transverse displacements for the aspect ratio of 4 and 10 are presented in Tables 1 and 2 for a simply supported beam subjected to sinusoidal load. The transverse displacement values by present theory using general solution and the values by closed form analytical solution of Ghugal and Shimpi [22] are identical. Present theory overestimates this value by $1.761 \%$ compare to exact solution for aspect ratio 4 and by $0.44 \%$ for aspect ratio 10. The results of higher order model by Lu and Liu and results of first order shear deformation theory using finite element solution by Maiti and Sinha [overestimate this value by $2.48 \%$ and $2.75 \%$ respectively for aspect ratio 4 . The higher model by Maiti and Sinha underestimates the value by $24.17 \%$ compared to the value of exact solution for aspect ratio 4 . The results of present solution, Shimpi and Ghugal (LTSDT), HOSTB5 of Manjunatha and Kant, Lu and Liu (HSDT) are closed to each other for aspect ratio 10, whereas the ETB underestimates the maximum transverse deflection by $43.62 \%$ for aspect ratio 4 and $11.25 \%$ for aspect ratio 10 and FSDT overestimates it by $2.89 \%$ compared to exact value for aspect ratio 4 and is in close agreement with the value of present theory for aspect ratio 10 . The graphical presentation of this displacement is shown in Fig. 2.

The transverse displacement results of fixed beam subjected to sinusoidal load are presented in Table 3 for aspect ratios 4 and 10. Compared to present theory; ETB underestimates the transverse deflection by $70.90 \%$, whereas FSDT overestimates the value by $0.873 \%$ for aspect ratio 4 . For aspect ratio 10, ETB underestimates the transverse deflection value by $38.25 \%$ and FSDT underestimates it by $0.175 \%$ when compared to the value given by present theory. The graphical presentation of this displacement is shown in Fig. 11. 


\subsection{Axial displacement $(\bar{u})$ :}

The results of axial displacement $\bar{u}$ are presented in Tables 1 and 2 for simply supported beam for aspect ratio 4 and 10, respectively. In case of simply supported beam, maximum $\bar{u}$ occurs at simply supported end $(x=0$ or $x=L)$ at $z= \pm h / 2$. The value of $\bar{u}$ given by present theory is higher by $10.21 \%$ compared to exact value. ETB and FSDT yield identical values of this displacement. ETB and FSDT underestimate the values by $15.56 \%$ and $3.16 \%$ for aspect ratio 4 and 10, respectively compared to exact values. The theories of Kant and Manjunatha and Lu and Liu overestimate this value by $6.40 \%$ and $3.19 \%$ respectively, for aspect ratio 4 when compared to exact values. The variation of this displacement through the thickness is graphically presented in Fig. 3 and Fig. 4 for aspect ratio 4 and 10 respectively, which shows the realistic variation indicating the effect of shear deformation on the deformation of transverse normal.

In the case of fixed beam, maximum $\bar{u}$ occurs at quarter span of the beam from the fixed end support and it diminishes at fixed ends and at the middle span of the beam. The graphical presentation of this displacement is shown in Figs. 12 and 13. At quarter span $(x=0.25 L)$ it shows considerable departure from the distributions given by ETB and FSDT at the same location. However, according to present theory, values of this displacement matches with the one given by ETB and FSDT at $(x=0.3758 L)$ from the support for aspect ratio 4 and at $(x=$ $0.3143 L$ ) for aspect ratio 10 .

\subsection{Axial stress $\left(\bar{\sigma}_{x}\right)$ :}

The results of maximum non-dimensional axial stress are given in Tables 1 and 2 for simply supported beam subjected to sinusoidal load for aspect ratio 4 and 10, respectively. ETB and FSDT yield the identical values for this stress. ETB and FSDT underpredict axial stress value by $20.83 \%$ and $4.51 \%$ at top compared to exact values for aspect ratio 4 and 10, respectively. The results by Kant and Manjunatha and $\mathrm{Lu}$ and Liu underestimate the axial stress by $2.32 \%$ and $6.94 \%$ compared to exact value for aspect ratio 4. For aspect ratio 10, Manjunatha and Kant underestimate the value by $0.605 \%$, whereas $\mathrm{Lu}$ and Liu overestimate it by $0.857 \%$ compared to exact value. The distributions of this stress are shown in Figs. 5 and 6 . Axial stress variation through the thickness shows the severe influence of shear deformation effect for aspect ratio 4 . (see Fig.5) as compared to the variation for aspect ratio 10.

The results of maximum non-dimensional axial stresses obtained by the present theory, ETB, FSDT are presented in Table 3 for fixed beam subjected to sinusoidal load for aspect ratio 4 and 10. The results of the maximum non-dimensional stress by present theory match to those of ETB and FSDT at $0.0745 \mathrm{~L}$ and $0.03937 \mathrm{~L}$ from the left end support for aspect ratio 4 and 10 respectively. Present theory yields very high value at the fixed support which may be attributed to high stress concentration at this end. These stresses are presented graphically in Figs.14 and 15 which show realistic variation of this stress indicating the effects of both shear deformation and stress concentration at the fixed end.

Latin American Journal of Solids and Structures 10(2013) $675-705$ 


\subsection{Transverse shear stresses $\left(\bar{\tau}_{x z}\right)$ :}

The transverse shear stresses are obtained directly by constitutive relation and, alternatively by integration of equilibrium equation of two dimensional elasticity and are denoted by $\left(\bar{\tau}_{z x}^{C R}\right)$ and $\left(\bar{\tau}_{z x}^{E E}\right)$ respectively. The transverse shear stress satisfies the stress free boundary conditions on the top $(z=-h / 2)$ and bottom $(z=+h / 2)$ surfaces of the beam when these stresses are obtained by both the above mentioned approaches.

The non-dimensional transverse shear stress values for simply supported beam subjected to sinusoidal load are presented in Table 1 and 2 for aspect ratio 4 and 10 respectively. Transverse shear stress value obtained by present theory according to constitutive relation is $10.40 \%$ higher for aspect ratio 4 and $5.63 \%$ higher for aspect ratio 10, compared to values obtained by equilibrium equations. The results obtained by present theory using constitutive relations are in good agreement with exact results, whereas the results obtained by equilibrium equations are in excellent agreement with those of exact elasticity solution. The higher order model by Maiti and Sinha underestimates the value by $10.87 \%$ compared to the exact value, whereas the value obtained by Vinayak et al. using higher order model is on higher side by $1.05 \%$ for aspect ratio 4 . The through the thickness variations of this stress using constitutive relation are shown in Figs. 7 and 8. and those given by equilibrium equation are given in Figs.9 and 10 .

The non-dimensional transverse shear stress values for fixed beam subjected to sinusoidal load are presented in Table 3 for aspect ratio 4 and 10. According to present theory, the transverse shear stress value by constitutive relation is $22.05 \%$ higher for aspect ratio 4 and $20.24 \%$ higher for aspect ratio 10 at $(x=0.2 L)$ compared to the corresponding values obtained by equilibrium equation. ETB and FSDT yield identical values of this stress at fixed end when obtained using equilibrium equation. In case of fixed beam, through the thickness distribution of $\bar{\tau}_{z x}^{E E}$, as shown in Figs. 18 and 19, given by present theory according to equilibrium equation shows the considerable deviation from the distributions given by ETB and FSDT at the fixed end $(x=0)$ with change in sign. The maximum negative value of this stress occurred at neutral axis and maximum positive value occurred at interface $(z=0$, centroidal axis). This anomalous behavior is attributed to heavy local stress concentration at this end. However, this behavior can not be captured by use of constitutive relation. The effect of stress concentration diminishes at other locations away from the fixed end (see Figs. 18 and 19). The graphical representation of transverse shear stress using equilibrium equation shows the effect of stretching bending coupling in the bottom layer, in which fiber orientation is $0^{0}$ that is along the $x$ - axis. This effect can not be captured by ETB and FSDT. Thus the present theory gives the realistic variation of this stress at the fixed end. The effect of variation of aspect ratio on through the thickness distribution of this stress is shown in Fig. 20. 


\section{CONCLUSIONS}

A layerwise shear deformation theory is used for the static flexural analysis of cross ply laminated (90/0) simply supported and fixed beams subjected to sinusoidal load. Euler-Bernoulli's and Timoshenko's classical theories are employed in a layerwise manner to obtain results. A general solution technique is developed for the static flexure of beams based on present theory. The results by present theory for simply supported beam subjected to sinusoidal load are validated by comparing with the results by exact solution. However, the exact solution for fixed-fixed beam subjected to sinusoidal load is not available; hence results are compared with those of EulerBernoulli and Timoshenko classical theories. The present theory is capable to capture the effect of stress concentration at fixed end and account for the shear deformation effect in un-symmetric cross-ply laminated beams. The results obtained by present theory are in good agreement with exact elasticity solution of laminated beam. The present theory can be applied to the laminated beams with various loading and boundary conditions by developing general solution of the problem.

\section{References}

[1] S. P. Timoshenko. On the Correction for Shear of the Differential Equation for Transverse Vibrations of Prismatic Bars. Philosophical Magzine, series 6(41):742-6, 1921.

[2] Y. M. Ghugal and R. P. Shimpi. A review of refined shear deformation theories for isotropic and anisotropic laminated beams. Journal of Reinforced Plastics and Composites, 20(3): 255-73, 2001.

[3] J. N. Reddy. Mechanics of laminated composite plates and shells: theory and analysis. $2^{\text {nd }}$ edition, Boca Raton, FL: CRC Press; 2004.

[4] I. Kreja. A literature review on computational models for laminated composite and sandwich panels. Central European Journal of Engineering, 1(1):59-80, 2011.

[5] T. Kant and B. S. Manjunatha. Refined theories for composite and sandwich beams with $\mathrm{C}^{0}$ finite elements. Computers and Structures, 33: 755-64, 1989.

[6] K. P. Soldato and P. Watson. A method for improving the stress analysis performance of one- and twodimensional theories for laminated composites. Acta Mechanica, 123:163-86, 1997.

[7] A. M. Zenkour. Transverse shear and normal deformation theory for bending analysis of laminated and sandwich elastic beams. Mechanics of Composite Materials and Structures, 6: 267-83, 1999.

[8] B. S. Manjunatha and T. Kant. Different numerical techniques for the estimation of multiaxial stresses in symmetric/unsymmetric composite and sandwich beams with refined theories. Composite Structures, 23: 61731993.

[9] D. K. Maiti and Sinha. Bending and Free Vibration Analysis of Shear deformable Laminated Composite Beams by Finite Element Method. Composite Structures, 29: 421-31, 1994.

[10] R. U. Vinayak, G. Pratap and B. P. Naganarayana. Beam Elements Based on a Higher Order Theory - I: Formulation and Analysis of Performance. Computers and Structures, 58: 775-89, 1996.

[11] K. H. Lo, R. M. Christensen, E. M. Wu. A Higher Order Theory for Plate Deformations, Part 1: Homogeneous Plates. ASME journal of Applied Mechanics, 663-68, 1977.

[12] J. Park and S. Y.Lee. A new exponential plate theory for laminated composites under cylindrical bending. Transactions of the Japan Society for Aeronautical and Space Sciences, 46 (152): 89-95, 2003.

[13] A. A. Khdeir and J. N. Reddy. An exact solution for the bending of thin and thick cross-ply laminated beams. Composite Structures, 37:195-203, 1997.

[14] M. Tahani, Analysis of laminated composite beams using layerwise displacement theories. Composite Structures, 79: 535-547, 2007.

Latin American Journal of Solids and Structures 10(2013) $675-705$ 
[15] D. Liu and X. Li. An overall View of Laminate Theories based on Displacement Hypothesis. Journal of Composite Materials, 30: 1539-61, 1996.

[16] X. Li and D. Liu. Zig-zag theories for composite laminates: Technical Note. AIAA Journal, 33: 1163-1165, 1995.

[17] U. Icardi. A three dimensional zig -zag theory for analysis of thick laminated beams. Composite structures, 52: 123-35, 2001.

[18] H. Arya, R. P. Shimpi and N. K. Naik. A zigzag model for laminated composite beams. Composite Structures, 56:21-4, 2002.

[19] J. N. Reddy and D. H. Robbins. Theories and Computational Models for Composite Laminates. Applied Mechanics Reviews, 47:147-169, 1994.

[20] X. Lu and D. Liu. An interlaminar shear stress continuity theory for both thin and thick Laminates. ASME Journal of Applied Mechanics, 59: 502-509, 1992.

[21] A. Catapano, G. Giunta, S. Belouettar and E. Carrera. Static analysis of laminated beams via a unified formulation. Composite structures, 94(1):75-83, 2011

[22] Y. M. Ghugal and R. P. Shimpi. A new Layerwise Trigonometric Shear Deformation Theory for Two Layered Cross-Ply Laminated Beams. Composites Science and Technology, 61: 1271-1283, 2001.

[23] G. M. Gere and S. P. Timoshenko. Mechanics of Materials: CBS Publishers. New Delhi. ${ }^{\text {st }}$ Indian edition. 407- 414, 1986.

[24] Y. M. Ghugal. A two-dimensional exact elasticity solution of thick beam. Departmental report No.1. Department of Applied Mechanics, Government Engineering College Aurangabad, India.1-96, 2006.

\section{Appendix}

The constants $A_{\mathrm{i}}$ and $B_{i}$ appeared in flexural rigidities $D, D_{1}, D_{2}$ and $D_{3}$ of governing differential equations and boundary conditions [Eqs. (15) through (19)] are defined as follows:

(a) Layer 1 integration constants $A_{1}, A_{2}, A_{3}, A_{4}$

$$
\begin{aligned}
& A_{1}=b h^{3} E^{(1)}\left[\frac{1}{24}+\frac{\alpha}{4}+\frac{\alpha^{2}}{2}\right] \\
& A_{2}=b h^{3} E^{(1)}\left\{C_{1}\left(\frac{1}{8}+\frac{\alpha}{2}\right)+C_{2}\left(\frac{1+2 \alpha}{\pi}\right)^{2}\left[1+\sin \left(\frac{-\pi \alpha}{1+2 \alpha}\right)\right]+\alpha C_{2}\left(\frac{1+2 \alpha}{\pi}\right) \cos \left(\frac{-\pi \alpha}{1+2 \alpha}\right)\right\} \\
& A_{3}=b h^{3} E^{(1)}\left\{\frac{C_{1}^{2}}{2}-2 C_{1} C_{2}\left(\frac{1+2 \alpha}{\pi}\right) \cos \left(\frac{-\pi \alpha}{1+2 \alpha}\right)+\frac{C_{2}^{2}}{4}\left[1+\left(\frac{1+2 \alpha}{\pi}\right) \sin \left(\frac{\pi \alpha}{0.5+\alpha}\right)\right]\right\} \\
& A_{4}=\frac{b h G^{(1)}}{4}\left(\frac{C_{2} \pi}{1+2 \alpha}\right)^{2}\left[1+\left(\frac{1+2 \alpha}{\pi}\right) \sin \left(\frac{-\pi \alpha}{0.5+\alpha}\right)\right]
\end{aligned}
$$


(b) Layer 2 integration constants $B_{1}, B_{2}, B_{3}, B_{4}$

$$
\begin{aligned}
& B_{1}=b h^{3} E^{(2)}\left[\frac{1}{24}-\frac{\alpha}{4}+\frac{\alpha^{2}}{2}\right] \\
& B_{2}=b h^{3} E^{(2)}\left\{C_{3}\left(\frac{1}{8}-\frac{\alpha}{2}\right)+\left(\frac{1-2 \alpha}{\pi}\right)^{2}\left[1+\sin \left(\frac{\pi \alpha}{1-2 \alpha}\right)\right]-\alpha\left(\frac{1-2 \alpha}{\pi}\right) \cos \left(\frac{-\pi \alpha}{1-2 \alpha}\right)\right\} \\
& B_{3}=b h^{3} E^{(2)}\left\{\frac{C_{3}^{2}}{2}+2 C_{3}\left(\frac{1-2 \alpha}{\pi}\right) \cos \left(\frac{-\pi \alpha}{1-2 \alpha}\right)+\frac{1}{4}\left[1-\left(\frac{1-2 \alpha}{\pi}\right) \sin \left(\frac{\pi \alpha}{0.5-\alpha}\right)\right]\right\} \\
& B_{4}=\frac{b h G^{(2)}}{4}\left(\frac{\pi}{1-2 \alpha}\right)^{2}\left[1-\left(\frac{1-2 \alpha}{\pi}\right) \sin \left(\frac{-\pi \alpha}{0.5-\alpha}\right)\right]
\end{aligned}
$$

(c) Constants $C_{1}, C_{2}, C_{3}$ are as follows:

$$
\begin{aligned}
& C_{1}=\left(\frac{E^{(2)}}{E^{(1)}+E^{(2)}}\right)\left\{\begin{array}{l}
\sin \left(\frac{-\pi \alpha}{1-2 \alpha}\right)-C_{2} \sin \left(\frac{-\pi \alpha}{1+2 \alpha}\right)+2 C_{2}\left(\frac{E^{(1)}}{E^{(2)}}\right)\left(\frac{1+2 \alpha}{\pi}\right) \cos \left(\frac{\pi \alpha}{1+2 \alpha}\right) \\
-2\left(\frac{1-2 \alpha}{\pi}\right) \cos \left(\frac{\pi \alpha}{1-2 \alpha}\right)
\end{array}\right\} \\
& C_{2}=\frac{G^{(2)}(0.5+\alpha) \cos \left(\frac{-\pi \alpha}{1-2 \alpha}\right)}{G^{(1)}(0.5-\alpha) \cos \left(\frac{-\pi \alpha}{1+2 \alpha}\right)} \\
& C_{3}=\left(\frac{E^{(1)}}{E^{(1)}+E^{(2)}}\right)\left\{\begin{array}{l}
-\sin \left(\frac{-\pi \alpha}{1-2 \alpha}\right)+C_{2} \sin \left(\frac{-\pi \alpha}{1+2 \alpha}\right)+2 C_{2}\left(\frac{1+2 \alpha}{\pi}\right) \cos \left(\frac{\pi \alpha}{1+2 \alpha}\right) \\
-2\left(\frac{E^{(2)}}{E^{(1)}}\right)\left(\frac{1-2 \alpha}{\pi}\right) \cos \left(\frac{\pi \alpha}{1-2 \alpha}\right)
\end{array}\right\}
\end{aligned}
$$

(d) Constants $D, \bar{D}, D_{1}, D_{2}, D_{3}, \bar{D}_{3}, \alpha$ are as follows:

$$
\begin{aligned}
& D=\left(A_{1}+B_{1}\right)=\bar{D} E^{(2)} b h^{3}, \\
& \bar{D}=\left(\frac{E^{(1)}}{E^{(2)}}\right)\left(\frac{1}{24}+\frac{\alpha}{4}+\frac{\alpha^{2}}{2}\right)+\left(\frac{1}{24}-\frac{\alpha}{4}+\frac{\alpha^{2}}{2}\right)
\end{aligned}
$$




$$
\begin{aligned}
& D_{1}=\left(\frac{A_{2}+B_{2}}{A_{1}+B_{1}}\right), D_{2}=\left(\frac{A_{3}+B_{3}}{A_{2}+B_{2}}\right), \\
& D_{3}=\left(\frac{A_{4}+B_{4}}{A_{2}+B_{2}}\right), \bar{D}_{3}=D_{3} h^{2} \\
& \alpha=\frac{1}{4}\left(\frac{E^{(2)}-E^{(1)}}{E^{(2)}+E^{(1)}}\right)
\end{aligned}
$$

(e) The constants $\lambda$ and $\eta$ appeared in Eq. (21) are defined as follows.

$\lambda^{2}=\frac{\beta}{\eta}, \eta=D_{2}-D_{1}$ with $\beta=D_{3}$ 